\title{
Functional Outcome of Hemorrhagic Transformation after Thrombolysis for Ischemic Stroke: A Prospective Study
}

\author{
Mariam Annan Marie Gaudron Jean-Philippe Cottier Xavier Cazals \\ Maelle Dejobert Philippe Corcia Philippe Bertrand Karl Mondon \\ Bertrand de Toffol Séverine Debiais
}

Stroke Unit, University Hospital Center of Tours, Tours, France

\section{Key Words}

Hemorrhagic transformation · Functional outcome $\cdot$ Ischemic stroke $\cdot$ Thrombolysis

\begin{abstract}
Background/Aims: Hemorrhagic transformation (HT) is usually taken into account when symptomatic, but the role of asymptomatic HT is not well known. The aim of our study was to evaluate the link between $\mathrm{HT}$ after thrombolysis for ischemic stroke and functional outcome at 3 months, with particular emphasis on asymptomatic HT. Methods: Our study was performed prospectively between June 2012 and June 2013 in the Stroke Unit of the University Hospital Center of Tours (France). All patients treated with intravenous thrombolysis were consecutively included. HT was classified on susceptibility-weighted imaging (SWI) with 3-tesla MRI at $7 \pm 3$ days after treatment. We evaluated functional outcome at 3 months using the modified Rankin Scale (mRS). Dependency was defined as an mRS score of $\geq 3$. Results: After 1 year, 128 patients had received thrombolytic therapy for ischemic stroke, of whom 90 patients underwent both 3-tesla MRI and SWI at day 7. Fifty-two had HT, including 8 symptomatic cases. At 3 months, $68 \%$ of those patients were dependent compared to $31 \%$ of patients without HT [OR 4.6 (1.9-11.4), $p=0.001$ ]. In asymptomatic HT, the rate was 62\% [OR 3.5 (1.48.9), $p=0.007]$, but did not reach significance after adjustment for stroke severity. Discussion: Our study found no statistically significant effect of HT on outcome after adjustment for initial stroke severity. However, the innocuousness of HT is not certain, and only few studies have already highlighted the increased risk of dependency. Using 3-tesla MRI with SWI allows us to increase the detection rate of small hemorrhage. Conclusion: HT after thrombolysis is very frequent on SWI, but the initial stroke severity is an important predictor to assess the role of HT for patient outcome.




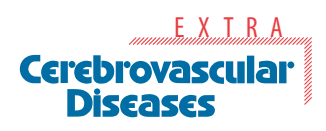

\section{Introduction}

Intracerebral hemorrhage (ICH) is a major complication of intravenous thrombolysis for acute ischemic stroke. It is now well established that symptomatic ICH severely impairs functional recovery and is associated with higher mortality [1]. In many stroke studies evaluating rt-PA, symptomatic ICH is usually defined as blood on follow-up brain imaging and is associated with neurologic deterioration of $\geq 4$ points on the NIHSS score. This definition is used in large randomized clinical trials, such as ECASS2 and SITS-MOST, and is considered one of the best methods to identify tPA hemorrhages that alter patient outcomes [2]. When ICH after thrombolytic therapy in acute ischemic stroke is not associated with such neurologic deterioration, it is defined as 'asymptomatic' and is rarely considered. Its clinical significance in neurologic outcome is not well known. Only few studies with controversial results exist on this topic. The question of the complete innocuousness of asymptomatic hemorrhage is debated.

The purpose of our study is to investigate the impact of hemorrhagic transformation (HT) on the 3-month disability and mortality in patients who benefited from intravenous thrombolysis for acute ischemic stroke, with particular emphasis on asymptomatic HT.

\section{Methods}

We prospectively analyzed all consecutive patients admitted to the Stroke Unit of the University Hospital Center of Tours (France) who were treated with rt-PA between June 2012 and June 2013. Only patients who had a 3-tesla MRI at the time of thrombolysis decision and on follow-up imaging were included in the analysis.

\section{Patient Data Collection}

The NIHSS score was evaluated at baseline, at day 1 after thrombolytic therapy, at day 7 and at 3 months. The modified Rankin Scale (mRS) score was evaluated at 3 months. Unfavorable outcome was defined as an mRS score of 3-6. Clinical data including age, sex, risk factors for stroke and treatments were recorded.

\section{MRI Data Collection}

All patients had a 3-tesla brain MRI (Verio Tim; Siemens AG, Erlangen, Germany), with a 12-channel antenna. The first MRI was performed under emergency conditions, as soon as possible and before $4.5 \mathrm{~h}$ after the beginning of symptoms. All MRIs were performed according to a standardized protocol including FLAIR, diffusion-weighted imaging (DWI), T2*-weighted gradient echo (GRE), susceptibility-weighted imaging (SWI), 3D time-of-flight and perfusion-weighted imaging. A second MRI including DWI, FLAIR, GRE and SWI sequences was performed at $7 \pm 3$ days after treatment. The presence of HT was evaluated on the second MRI, on SWI, according to the ECASS classification and classified as hemorrhagic infarction type 1 or 2, or parenchymal hematoma type 1 or 2 . According to the ECASS2 definition for clinically important hemorrhage, we defined symptomatic HT when any ICH was observed on the follow-up imaging and associated with an NIHSS score worsening of $\geq 4$ points. Asymptomatic HT was defined as any HT on follow-up MRI without any neurologic deterioration or a neurologic deterioration in the NIHSS score of $\leq 3$ points between baseline (or the lowest NIHSS score) and day 7 (or the highest NIHSS score).

This study was approved by our Institutional Ethics Committee. 

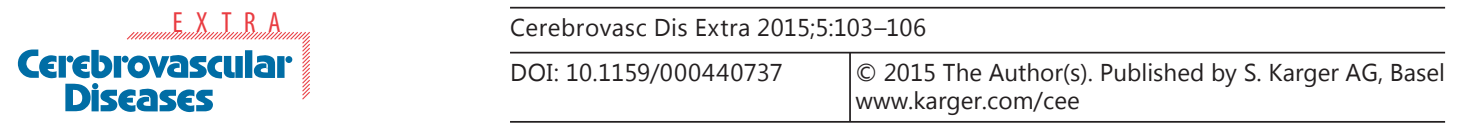

Annan et al.: Functional Outcome of Hemorrhagic Transformation after Thrombolysis for Ischemic Stroke: A Prospective Study

Table 1. Baseline characteristics of patients according to ICH status and functional outcome status ${ }^{1}$

\begin{tabular}{|c|c|c|c|c|c|c|}
\hline Characteristics & $\mathrm{HT}+(\mathrm{n}=52)$ & HT- $(n=38)$ & $\mathrm{p}$ & $\begin{array}{l}\text { mRS score of } \\
0-2(n=56)\end{array}$ & $\begin{array}{l}\text { mRS score of } \\
3-6(n=33)\end{array}$ & $\mathrm{p}$ \\
\hline Age, years & $74.3 \pm 11.4$ & $70.1 \pm 17.6$ & 0.18 & $70.1 \pm 14.8$ & $76.7 \pm 12.6$ & 0.035 \\
\hline Males & $36(69.2)$ & $19(50)$ & 0.07 & $35(62.5)$ & $19(57.6)$ & 0.65 \\
\hline Time to treatment, min & $197 \pm 46$ & $193 \pm 51$ & 0.66 & $193 \pm 52$ & $198 \pm 42$ & 0.66 \\
\hline Systolic blood pressure, $\mathrm{mm} \mathrm{Hg}$ & $159 \pm 24$ & $151 \pm 25$ & 0.11 & $157 \pm 26$ & $154 \pm 22$ & 0.64 \\
\hline Pretreatment NIHSS & $13 \pm 7$ & $8 \pm 5$ & $<0.01$ & $8 \pm 6$ & $16 \pm 7$ & $<0.01$ \\
\hline Hypertension & $35(67.3)$ & $25(65.8)$ & 0.88 & $35(62.5)$ & $25(75.8)$ & 0.20 \\
\hline Diabetes mellitus & 7 (13.5) & $6(15.8)$ & 0.76 & $8(14.3)$ & $4(12.1)$ & 0.77 \\
\hline Dyslipidemia & $17(32.7)$ & $15(39.5)$ & 0.51 & $20(35.7)$ & $13(39.4)$ & 0.73 \\
\hline Glucose at baseline, g/l & $1.35 \pm 0.32$ & $1.22 \pm 0.29$ & 0.04 & $1.26 \pm 0.31$ & $1.32 \pm 0.30$ & 0.34 \\
\hline Leukoaraiosis & $25(48.1)$ & 11 (28.9) & 0.07 & $18(32.1)$ & $17(51.5)$ & 0.07 \\
\hline Antithrombotic drugs & $24(46.2)$ & $17(44.7)$ & 0.89 & $23(41.1)$ & $18(54.5)$ & 0.22 \\
\hline
\end{tabular}

Values are mean \pm SD or n (\%). HT $+=$ Presence of HT; HT- = absence of HT; mRS score of 0-2 = 'good outcome'; mRS score of 3-6 = 'bad outcome'.

${ }^{1}$ Evaluated using mRS.

\section{Statistical Methods}

Statistical analyses were performed with SSPS software (version 18.0.0). Results are expressed as means and standard deviations for qualitative variables, and absolute values and percentages for qualitative variables. Comparisons were calculated with the $\chi^{2}$ test for qualitative variables and with the Student $t$ test for quantitative variables. The CochranMantel-Haenszel test was used to calculate the adjusted OR.

\section{Results}

After 1 year, a total of 128 patients had received thrombolytic therapy for ischemic stroke, of whom 90 patients underwent both 3-tesla MRI and SWI (pretreatment and control MRI at day 7). One of them was lost to follow-up at 3 months. Baseline characteristics of patients with and without HT and with good and bad outcomes are shown in table 1 . At the 3-month evaluation, dependency (mRS score 3-6) was present in 34 of 52 patients who showed HT (68\%) on MRI at day 7. Twelve (31.6\%) of 38 patients without HT were dependent. This difference reached significance [OR $4.6(1.9-11.4), \mathrm{p}=0.001]$. All patients with symptomatic ICH $(n=8)$ were dependent compared to $46.9 \%$ patients ( 38 of 81 patients; $p=0.004$ ) without HT or nonsymptomatic ICH. If we consider only patients with asymptomatic hemorrhage ( $\mathrm{n}=42), 26(62 \%)$ had a bad outcome at 3 months. This difference still reaches significance [OR 3.5 (1.4-8.9), $\mathrm{p}=0.007$ ]. The main confounding variable was the baseline NIHSS score. When considering asymptomatic HT, the adjusted OR for bad outcome was $0.96(0.25-$ 3.63).

\section{Discussion}

In this study, a high rate of HT was found using systematic 3-tesla MRI at day 7 after thrombolysis. Our study found that HT is associated with bad outcome; however, after adjustment for the baseline NIHSS, we could not statistically prove that asymptomatic HT is 
associated with a higher rate of dependency at 3 months. The innocuousness of HT has previously been debated in a couple of studies. Molina et al. [3] concluded that there was a higher rate of good outcome in patients with asymptomatic hemorrhage and explains this result by the fact that it could reflect recanalization. Nevertheless, this has not been confirmed by other studies: more often, small but significantly higher rates of bad outcome were found in patients with asymptomatic hemorrhage $[4,5]$. Nevertheless, the literature is sparse, especially about postthrombolysis ICH. In our study, we performed a systematic MRI with SWI, at day 7 after stroke, thus increasing the detection rate of asymptomatic hemorrhage. However, the small population sample is a clear limit of our study. Besides, it is necessary to note that stroke severity is a clearly important factor to consider in outcome, and is also associated with HT [6]. The interrelationship of those two factors does not facilitate the determination of the intrinsic effect of hemorrhage on outcome. Probably a higher number of patients would be necessary. The question is still clinically pertinent, since the rate of HT is very high when using a high-performance systematic imaging method.

Our study also found an increased risk of HT due to the baseline glucose rate, which has been described elsewhere [7].

\section{Conclusion}

Systematic MRI with SWI reveals a high rate of postthrombolysis HT, associated with a bad functional outcome. After adjustment for initial stroke severity, no statistically significant impact of asymptomatic HT on outcome could be proven.

\section{Disclosure Statement}

The authors have no sponsorship nor funding arrangements relating to the research to disclose. None of the authors has any conflicts of interest.

\section{References}

1 Strbian D, Sairanen T, Meretoja A, Pitkäniemi J, Putaala J, Salonen O, et al; Helsinki Stroke Thrombolysis Registry Group: Patient outcomes from symptomatic intracerebral hemorrhage after stroke thrombolysis. Neurology 2011;77:341-348.

-2 Rao NM, Levine SR, Gornbein JA, Saver JL: Defining clinically relevant cerebral hemorrhage after thrombolytic therapy for stroke: analysis of the National Institute of Neurological Disorders and Stroke tissue-type plasminogen activator trials. Stroke 2014;45:2728-2733.

-3 Molina CA, Alvarez-Sabin J, Montaner J, Abilleira S, Arenillas JF, Coscojuela P, et al: Thrombolysis-related hemorrhagic infarction: a marker of early reperfusion, reduced infarct size, and improved outcome in patients with proximal middle cerebral artery occlusion. Stroke 2002;33:1551-1556.

4 Lei C, Wu B, Liu M, Chen Y: Asymptomatic hemorrhagic transformation after acute ischemic stroke: is it clinically innocuous? J Stroke Cerebrovasc Dis 2014;23:2767-2772.

5 Park JH, Ko Y, Kim W-J, Jang MS, Yang MH, Han M-K, et al: Is asymptomatic hemorrhagic transformation really innocuous? Neurology 2012;78:421-426.

6 Kent DM: In acute ischemic stroke, are asymptomatic intracranial hemorrhages clinically innocuous? Stroke 2004;35:1141-1146.

7 Mazya M, Egido JA, Ford GA, Lees KR, Mikulik R, Toni D, et al; for the SITS Investigators: Predicting the risk of symptomatic intracerebral hemorrhage in ischemic stroke treated with intravenous alteplase: safe Implementation of Treatments in Stroke (SITS) symptomatic intracerebral hemorrhage risk score. Stroke 2012;43: 1524-1531. 